\title{
Aciduria glutárica tipo I: una encefalopatía metabólica extrapiramidal
}

\author{
Mónica Troncoso Sch. ${ }^{3}$; Fernando Novoa S. ${ }^{3}$; Marta Colombo C. ${ }^{2}$; \\ Ledia Troncoso A. '; Lautaro Badilla O.
}

\section{Glutaric aciduria, type I}

\begin{abstract}
Three chilfren [two girls] aged lour, six and Iwo years with rype 1 glutaric aciduria are described. Their developmen wos norral until the second half of trie first year of life, when they had acule diarhea, acidosis. coma and seizures. Afterwards they lost acquired mental ond motor abilities and showed abnormal movements, porticularly dystoria. Brain CAT scan and NMRI demostrated in all them signs of fronlal, temporct, caudale nucleus and putomer.'s atrophy. These three chuldren excreted increosed amounts of glutaric acid, 3-hydroxiglutaric ocid and glutaconic acid and decreased activity of the enzime glutayil Coo dehidrogenase was recorded from their cultured fibroblasts, corlirming the diognosis of glutaric acidurio typel.
\end{abstract}

[Kay words: basal ganglia, bran diseases, dystonia, glutaric aciduria, lype l.]

La aciduria glutárica tipo I es un error congénito del metabolismo de los aminoácidos, de herencia autosómica recesiva, causada por la ausencia o marcada reducción de la actividad de la glutaril $\mathrm{CoA}$ dehidrogenasa, enzima que actúa en la vía oxidativa de la lisina, hidroxilisina y triptófano'.

Esta entidad fue descrita por Goodman y col en $1975^{2}$; generalmente aparece durante el primer año de vida y se manifiesta fundamentalmente de dos formas: una encefalopatía aguda con acidosis metabolica, después de la cual aparecen distonía o coreoatetosis y deterioro del desarrollo psicomotor, o bien una enfermedad subaguda, con retraso psicomotor progresivo. seguido de síntomas extrapiramidales'. En la tomografía axial computadorizada (TAC) de cerebro y la resonancia nuclear magnética (RNM) se registra atrofia frontotemporal bilateral $y$, con menor frecuencia, de los núcleos caudados y lenticulares; se han descrito también cambios difusos en la sustancia blanca ${ }^{3-6}$. El diagnóstico

I. Servicio Neurología Infantil, Hospilal Clínico San Borja-Artiasán.

2. Unidad Neuropsicología INTA, Universidad de Chile.

3. Servicio Neurorradiología Instituto de Neurocirugía Dr. Asenio. se confirma demostrando los ácidos glutárico, 3-hidroxiglutárico y glutacónico en orina, y la deficiente actividad de la enzima aludida en cultivos de fibroblastos y leucocitos!.

El propósita de esta publicación es describir tres niños con esta afección, para enfatizar la conveniencia de incluirla entre las causas de encefalopatías que cursan con movimientos anormales en la niñez.

\section{Casos clínicos}

1. Varón de 4 años, padres no consanguíneos, gestacion y parto normales; sin antecedentes familiares. Al nacer: peso $3950 \mathrm{~g}$. talla $51,5 \mathrm{~cm}$, perfmetro craneal $36.5 \mathrm{~cm}$, apgar 5 al minuto y 9 a los 5 minutos. Desartollo normal hasta los 8 meses, cuando sufrió un episodio de diarrea aguda, deshidratación, acidosis metabólica severa, convulsiones genecalizadas y alteración de la conciencia. Una vez recuperado, su desarrollo psicomotor involucionó, hasia perder el control cefálico y apareció un intenso síndrome extrapiramidal caracterizado por distonía generalizada oromandibutar, retrocolis, pronación de las extremidades superiores, hiperlordosis, opistótonos, hiperextensión de las extremidades inferiores. Hemograma, uremia, glicennia, uricemia, velocidad de sedimentacion eritrocitaria, aminoacidemia aminoaciduria. ceruloplasmina, examen completo de orina, amonemia, examen líquido cerebro espinal (LCR), electroencefalogramas, conducción nerviosa y potenciales evocados auditivos y visuales resultaton potma- 
les en los dos años siguientes. La lactoacidemia, también normal en varias oportunidades, estaba levemente aumentada al final de ese períado. A los tres afios de edad, la TAC y RNM del cerebro mostraban dilatación temporosilyiana bilateral, degeneración y atrofia de los núclcos caudados y lenticulares y altetación difusa de la sustancia blanes (figura 1 ). En la oruna se registró eliminación anorinal de úcido 3-hidroxiglutarico. La actividad de Io enzima glutaryl CoA dehidrogenasa en cultivo de fibroblasios cstaba muy disminuida. Se inicis tratamiento con dieta hipoproteica. pobre en lisina y triptofano: riboflavina (100 ing - dia), tiamina. carnitina (100 mg • kg - dis), oligoelementos $y$ tríhexifenidilo (como tratamiento sintomático de la dislonia), con escasa respuesta. Por dificultad en la deglución fue necesario hacerle gastrostomia. Al caho de un año de seguimiento su estado neurológico no ha variado.

2. Niña de 6 años de edad, gestación y parto normales, padres no consanguíneos. Al nacer: peso $3100 \mathrm{~kg}$, talla 52 ctn. perímetro craneal $36 \mathrm{~cm}$, apgar 9 . Desartollo normal hasta los 6 meses de edad en que sufrió diarrea, alteraciones de contiencia. convulsiones tonicoclónicas y distonía generalizadas, hipoglicenia, hiperamonetnia, hiperJactatemia. axidosis metabólica. cuerpos cetónicos en otinu. sin alteraciones del LCR. Posteriormente evolucionó con intensa distonía generalizada, mayor en el hemicuerpo derecho: disquinesias oromandibulares de escasa respuesta a] tribexifenildilo; infecciones respiralorias reiteradas, sin descompensaciones metaboblicas. En la TAC cerebral, a tos cinco años de edad. se registró dilatación bilateral de los espacios snbaracnoideos temporosilvianos, atrofia de los núcleos caudados y lesiones simétricas de los lenticulares. En la orina se encontrô gran cantidad de ácido glutárico y menor de ácjdos 3-hidroxiglutárico y glutacónico. La actividad de la glutaril CoA dehidrogerasa en los fibroblastos estaba muy disminuida. Actualmente en tratomiento con dieta hipoproteica. riboflavina, carnitina. además, en forma intermitente, tribexifenidilo y baclofeno con escasa respuesta de sus síntomas distónicos. Después de un año de tratamicnto su condición neurológica no ha variado, tiene buen contacto social, no kabla, no bay ajteración de pares craneanos, sufre un síndrome pseudpbulbar, no controla la cabeza y su perímetro craneal es $54 \mathrm{~cm}$.

3. Nita de dos ar̃os de edad, padres no consanguineos. Gestación y parto normales. Al nacer: peso $3100 \mathrm{~g}$, talla $48 \mathrm{~cm}$, CC $36 \mathrm{~cm}$, apgar 9 . Sana con desarrollo normal hasta el año de edad, momento en el cual sufrió diarrea. vómitos y fiebre, alteración de la conciencia, conyulsiones del hemicuerpo derecho. distonía del euelto, tronco y extremidades derechas, perdiendo todas las habilidades adquiridas. Los exámenes de laboratorio mostraban intensa acidosis metabólica, y en la TAC cerebral se observaba dilatación temporosilviana bilateral y tenue hipodensidad de los núcleos grises centrales. Un mes después de iniciar este episodio el perínetro craneano era $49 \mathrm{~cm}$. no controlaba cabeza y tronco, escaso contacto social, irritable, difi-
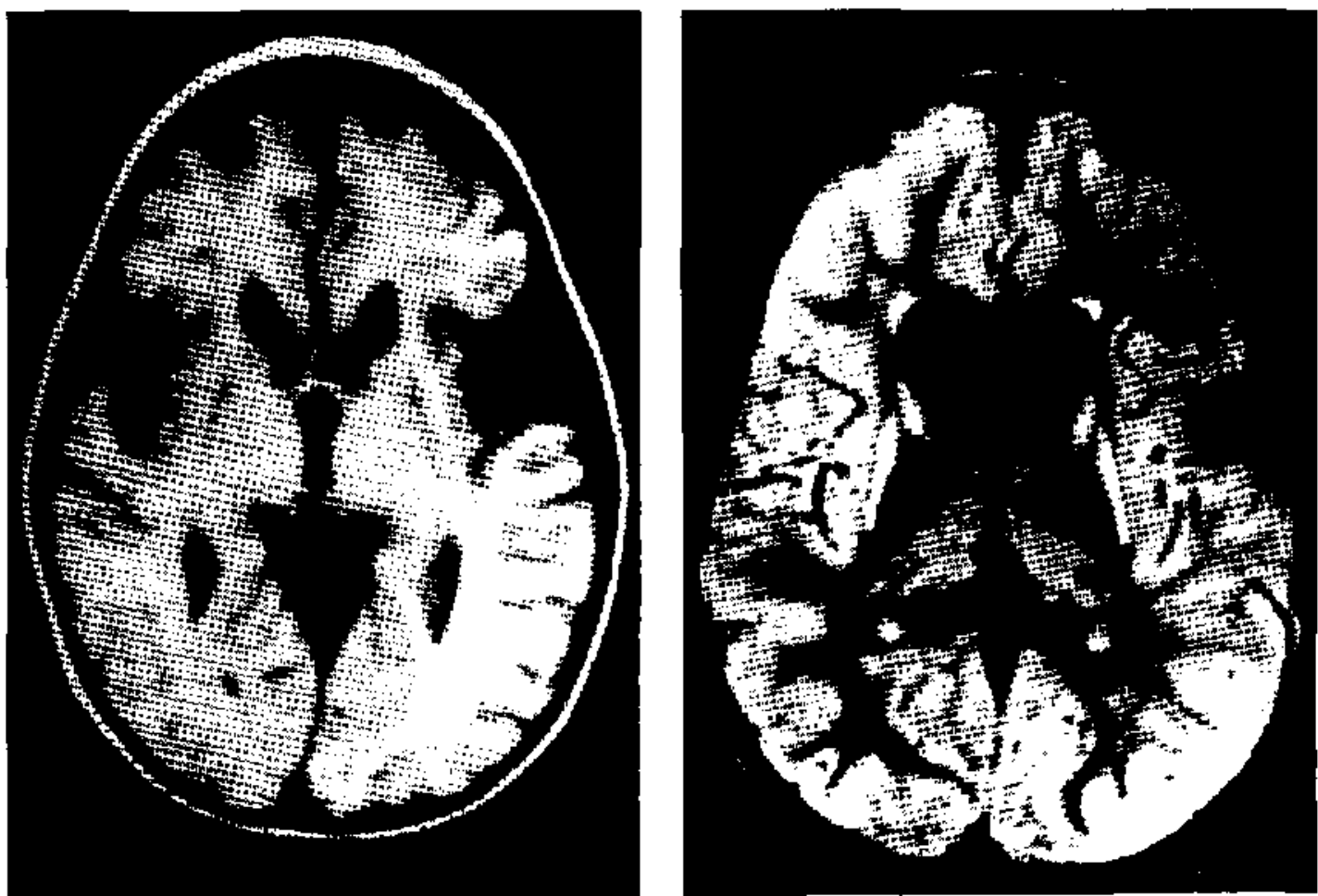

Figura 1: Inágenes del caso 1 por resonancia nuclear magnética. a); dilatación bilaleral de los valles sitvianos, atrofia de los núcleos caudados; b) degeneración simétrica característica de les núcleos lenticulares, compromiso difuso de sustancia blanca. 
cultad para deglutir, distonía del cuello, tronco y extre. midades derechas, sin alteraciones de los pares craneanos. Había graodes cantidades de ácidos glu1árico, 3hidroxiglutárico y glutacónico en la arina y franca disminución de la actividad de la glutaril CoA debidrogenasa en el cultivo de fibroblastos. Comenzó tratamiento con dieta pobre en lisina, hidroxilisina y triprofano, más riboflavina $100 \mathrm{mg} \cdot$ día, carnitina $100 \mathrm{mg} \cdot \mathrm{kg} \cdot \mathrm{dia}$, sin variar su estado neurológico después de un año de seguimiento. A los 4 meses de evolución. en la TAC apareción marcada dilataciún de los cuernos frontales de los ventrículos laterales. por atrofia de los núcleos caudados y un mes más tarde. en la RNM se confirmó lo anterior y, ademís, alteraciones difusas de la sustancia blanca y los núcleos lenticulares.

\section{Comentario}

La aciduria glutárica tipo I es un error congénito del metabolistmo de los aminoácidos, poco trecuente, que se expresa generalmente durante el primer año de vida, de inicio clínico agudo o insidioso y curso estático, progresivo o recurrente?

La forma más frecuente de presentación es la de estos pacientes, con desarrollo normal hasta el segundo semestre de vida y que sufren un trastorno agudo tóxico, metabólico, infeccioso, con acidosis metabólica, interpretado generalmente como "encefalitis", después del cual quedan delerioro psicomotor y síntomas extrapiramidales que dominan las manifestaciones clínicas, con gran incapacidad, que no se recupera ${ }^{7-9}$.

Otra forma, menos frecuente, de presentación es la instalación gradual de los síntomas en los primeros años de vida, apareciendo lentamente retraso psicomotor, a veces convulsiones. con o sin acidosis metabólica recurrente, agregándose luego los síntomas extrapiramidales, más frecuentemente distonía, pero también coreoatctosis ${ }^{7.9-12}$, que resultan invalidantes. Uno de los signos clínicos frecuentes es la macrocefalia, que puede encontrarse tan precozmente como en el período de recién nacido. Nuestros pacientes tenían, al nacer, perímetros craneanos cercanos al percentil $98 \mathrm{y}$, al ingresar a control, más de 2 desviaciones estándar sobre lo esperado. De la misma forma que en nuestros casos, la capacidad intelectual se deteriora relativamente poco, de manera que el retardo mental no siempre está presente, en cambio generalmente existe hipotonía, y los signos piramidales, de intensidad variable, son menos constantes $7,8,10.11,13,14$.
La intensidad de las manifestaciones de la afección varía de un caso a otro, siendo, los más leves, los de niños con aprendizaje escolar lento o leve hipotonía con torpeza motora, en los cuales el diagnóstico se hizo después de confirmar la enfermedad en hermanos o parientes cercanos, incluyendo uno identificado a la eda de 10 años, aún libre de síntomas ${ }^{7.8}$. En el otro extremo hay niños intensamente dañados, con gran distonía o coreoatetosis, retardo mental y cuadriparesia espástica, desde muy temprana edad. Estos niños con frecuencia son diagnosticados como parálisis cerebral. Los exámenes de laboratorio generalmente son normales en los períodos intercríticos. La acidosis metabólica no siempre está presente $y$, at igual que la hiperlactatenua, habitualmente se encuentran en los períodos de descompensación, relacionados -por lo general- con una afección infecciosa; puede haber hipoglicemia, hiperamonemia y cuerpos cetónicos en orina. En algunos casos se ha encontrado disminución de la carnitina en sangre, que -a veces- se ha recuperado con la administración de ésta. El estudio de aminoácidos generalmente es normal. Aunque la clínica orienta al diagnóstico se debe buscar ácidos glutárico. 3-hidroxiglutárico y -con menor frecuenciaglutacónico, en orina y sangre para confirmarlo -como en los casos que se comentan-e, idealmente, estudiar la actividad enzimática en cultivo de tejidos para certificarlo. Cabe señalar, sin embargo, que la actividad residual de la enzima no guarda relación con la severidad de los síntomas ${ }^{3.7-11}$.

Así como las manifestaciones clínicas orientan hacia al diagnóstico, en la TAC y la RNM se registran alteraciones bastante características. Uno de los signos más tempranos es el aumento del espacio subaracnoideo temporosilviano, incluso en pacientes asintomáticos, hecho que corespondería a atrofia del tejido nervioso, aunque algunos piensan que podría tratarse de quistes aracnoidales ${ }^{16}$. Otra imagen característica, aunque no siempre presente, es la dilatación de los cuernos frontales de los ventrículos laterales, por atrofia de los núcleos caudados y degeneración de los lenticulares ${ }^{3-8,10,15}$. En nuestros pacientes 1 y 2, la TAC y la RMN, años después de haber iniciado los síntomas, ya registraron las dos alteraciones recién descritas. En cambio, en el tercer paciente, la TAC realizada a los cinco días de la primera descompen- 
sación metabólica sólo mostraba aumento de los espacios subaracnoideos temporosilvianos y tenue hipodensidad de los núcleos grises centrales (que podría sugerir necrosis estriatal), pero cuatro meses después se observó desaparición de los caudados, lo que apoyaría que esta imagen corresponde a atrofia de estos núcleos de la base. Otros signos observados son hipodensidades de la sustancia blanca en la TAC e hiperintensidad en modo $\mathrm{T} 2$ de la $\mathrm{RNM}^{3-7}$, los que también pudimos comprobar en nuestros pacientes. La secuencia de aparición de las alteraciones en estas imágenes sugieren que el aumento del espacio subaracnoideo temporosilviano, el signo más precoz, pudiera ser incluso congénito y que posteriormente, con la crisis metabólica, sobreviene el compromiso de la sustancia blanca y los núcleos grises centrales.

El tratamiento de estos pacientes incluye restricción de las proteinas de la dieta, que debe ser escasa en lisina y triptofano, ambos precursores del ácido glutárico, con lo que disminuye la excreción urinaria de este último, aunque muchas veces sin efecto clínico sobre el paciente ${ }^{I I}$. Con la riboflavina, vitamina cofactor de la glutaril CoA dehidrogenasa, se intenta aumentar la actividad residual de la enzima, con resultados variables, desde ninguno a alguna mejoría clínica o no progresión de los síntomas ${ }^{8-12}$ : nuestros casos sólo han experimentado muy discreta mejoría, aunque sin progresión de los síntomas. La carnitina ha sido utilizada para estimular la formación y excreción de acilcarnitina de cadena corta principalmente glutarilcarnitinal?, 18. En algunos casos se obtuvo un aumento de la excreción urinaria de ácido glutárico y modesta respuesta clínica ${ }^{8}$. También estaria indicada como suplemento en pacientes en que su concentración plasmática se encuentra disminuida. Como el ácido glutárico puede inhibir la síntesis de acido gamma aminobutírico (GABA) y hay disminución de éste en el cerebro de pacientes afectados, se ha administrado un análogo del GABA, baclofeno, sin una clara respuesta ${ }^{8.10,17}$. Otro tanto ha sucedjdo con el ácido valproico 9 . En algunos casos, en coincidencia con el tratamiento descrito, se ha observado detención del avance de los síntomas, pero la mejoria, si ocurre, es leve.

El proceso físiopatológico causante de esta encefalopatía distónica está probablemente asociado con el metabolismo de los neutrotrans- misores, específicamente con el GABA y el glutamato. En el cerebro de dos niños que fallecieron con esta enfermedad se demostró niveles elevados de ácido glutárico en el cortex frontal y ganglios basales, muy baja actividad de la enzima ácido glutámico descarboxilasa (GAD) en los ganglios basales y la sustancia nigra y extremadamente bajas concentraciones de GABA en los núcleos caudado y putamen ${ }^{11} 19$. Puesto que la glutámico descarboxilasa es inhibida por el ácido glutárico in vitro, la marcada reducción del GABA en el caudado y putamen sugieren deficiencia de la actividad de GAD antes de la muerte en áreas en que el ácido glutárico estaba aumentado 11,15

En la anatomía patológica de algunos pacientes fallecidos se ha encontrado desde cambios mínimos en los cuerpos estriados, hasta intensa pérdida de neuronas y extensa gliosis en los núcleos caudados y putamen ${ }^{11}$ 19. Parece que el striatum es el más afectado por la neurotoxicidad de esta enfermedad, explicando que los movimientos involuntarios anormales constituyan uno de los síntomas predominantes. El daño cerebral causado por la deficiencia enzimática o la acumulación de sustratos puede ocurrir, al menos en ciertos casos. algún tiempo antes de las manifestaciones clínicas, como lo sugieren los hallazgos en la TAC y la RMN de niños asintomáticos, con escasez comprobada de la enzima.

El diagnóstico prenatal es posible, determinando la concentración de ácido glutárico en el líquido amniótico y la actividad de la glutaril CoA dehidrogenasa en cultivos de células amnióticas o en vellosidades coriales durante el primer trimestre del embarazo ${ }^{20}$. Dado el considerable riesgo de que la enfermedad se manifieste con síntomas muy invalidantes, es fundamental dar consejo genético a la familia.

Es necesario descartar afecciones metabólicas, como la aciduria glutárica tipo I y otros, en los nifios que sufren encefalopatías o parálisis cerebral de causa poco clara, lo que permitirá tratamientos más adecuado, evitar la progresion de los síntomas y ofrecer consejo genético.

\section{Resumen}

Se describen tres niños, uno varón, de 4,6 y 2 años de edad, afectados de aciduria glutárica 
tipo I. Su desarrollo tue normal hasta la segunda mitad del primer año de vida, cuando sufrieron alteración de conciencia y convulsiones, seguidas de pérdida de las habilidades adquiridas, distonía y movimientos anormales. La tomografía axial y resonancia nuclear magnética de cerebro mostraron atrofia frontotemporal y de los núcleos caudados y putámenes. Había gran cantidad de ácidos glutárico, 3-hidroxiglutárico y glutacónico en orina y actividad disminuida de la enzima glutaril CoA dehidrogenasa en cultivas de fibroblastos de los tres niños, confirmándose así el diagnóstico de esta afección metabólica.

(Palabras clave: aciduria glutárica tipo I, distonía, enfermedades cerebrales, enfermedades de los núcleos basales.)

\section{Agradecimientos}

A Mireya Fernández, bioquînica del INTA. U, de Chile, por los cultivos de fibroblastos. A Stephen Goodman MD, U. de Colorado. EUA, por los and́lisis de ácidos orgánicos y actividad enzimática en fibroblastos. A la nutrícionista Verónica Comejo y a la médico Ena Ratmann. del IN'TA. por el manejo de los pacientes. A los médicos Daniel Villalobos y otros del Servicio de Dermatologia, Hospital San Borja-Artarán, por las biopsias de piel.

\section{Referencias}

1. Gordman $S$, Freman F: Organic acidemias due to the defects in Lysina oxidation: 2-Ketoadipic acidemia and glutaric acidemia. En: The metabolic and molecular bases of inherited disease. Chapter 42 . Vol. I. Scriver Ch., Beaudet A, Valle D (eds.) McGraw-Hill, Inc. New York. London, Madrid, Tokyo, 1995; 1451-1477.

2. Goodntut SI, Markey SP. Mue PG, Miles BS. Teng $C C$ : Glutaric acidurin: a "new" disorder of amino acid inetabolism, Biochem Med 1975; 12; 12-21.

3. Yuger J. McClariy B, Seshia S: CT-scan findings in an infant with glutaric aciduria type I. Dev Med Child Neurol 1988; 30; 808-811.

4. Altman $N$. Rovira $M$, Bauer $M$ : Glutaric Aciduria type l: MR findings in 1wo cases. AJNR 1991; 12; 966-968.
5. Barkovich $A J$ : Metabolic and destructive brain disorders. In: Pediatric Neuroimaging. Raven Press N.Y. 1990; 35-75.

6. Wolpert S, Burnes P: Metabolic and degenerative disorders. In: MRI in Pediatric Neuroradiology. Ed. Mosby Year Book, INC, 1992; 121-150.

7. Hawarth J, Booth F, Chudley A, et at: Phenotypic variability in glutatic aciduria type I: report of fourteen cases in five Canadian Indian kindreds. J Pediatr 1991; 118 : 52-8.

8. Amir $N$, Et-Peleg $O$. Shalev $R$, Christensen $E$ : Glutaric aciduria type I: clinical heterogeneity and neuroradiologic features. Neurology $1987 ; 37 ; 1654-1657$

9. Whetan D. Hill R, Ryan E. Spate M: L-Glutaric acidemia: investigation of a patient and his family. Pediatrics 1979: 63: 88-93.

10. Bergman t, Finegold D, Garmer $C$, et al.: Acute profound dystonia in Infants with Glutaric acidemia. Pediatrics 1989; 83, 2: 228-234

11. Leibel $R$. Shih V, Goodman S: Glutaric acidemia: a metabolic disorder causing progressive choreonthetosis. Neurology 1980; 30; 1 \$63-1 168 .

12. Lipkin P. Roe Ch. Goodman S. Batshaw M: A case of glutaric acidemia type 1: Effect of riboflarin and carnitina. J Pediatr 1988; vol. 112, 1; 62-65.

13. Iafolla A, Kahter S: Megalencephaly in the neonatal period as the initial manifestation of glutaric aciduria rype I. J Pediatr 1989: 114: 1004-1006.

14. Hoffman G. Trejz F. Barth P, et al.: Macrocephaly: an important indication for organic acid analysis. J Inher Metab Dis 1991: 14: 329-332.

15. Amir N, El-Peleg O, Shalev $R$, Christensen E: Glutaric aciduria type I: enzinatic and ceuroradiologic investigations of tw kindreds. I Pediatr 1989; 114: 983-989.

16. Hald J. Nakstad P. Skjeldal O. Stromme P: Bilateral arachnoid cysts of the temporal fossa in fout children with glutaric aciduria type I. AJNR 199!; 12: 407 409

17. Brandt NJ. Gregersen N. Christensen $E_{1}$ Grn $I H_{1}$ Rusmussen $K$ : Treatment of glutaryl CoA dehydrogenase deficiency (glutaric aciduria): experience with diet, ribotlavin and GABA analogue. 3 Pediatr 1979 : 94: 669-73.

18. Seccombe $D W$, James L, Booth F: L-Carnitina treatment in glutaric aciduria type I. Neurology 1986 ; 36: $264-267$

19. Goodman SI, Norenberg MD. Shikes RH, Breslich DJ. Moe $P G$ : Glularic aciduria: biochemical and morphologic considerations. J Pediatr 1977; 90: 746-750.

20. Goodman SI, Galleg DA, Pullin CJ, et ul.: Antenatal diagnosis of glutaric acidemia. Am J Human Genet 1980; 32: 695-699. 
Research Officer", para regresar al país, donde prosigue, $y$ con idéntico brío, las actividades de enseñanza pediátrica continua. Con este propósito, cre6 en 1982 la Fundación de Docencia en Salud del Niño y de la Familia (FUDOC), institución con personalidad jurídica y que hasta la fecha ha realizado una extraordinaria labor, en el desarrollo de Cursos de Actualización en Pediatría Integral, tanto en Santiago como en provincias. Para ello se ha contado con el apoyo del Ministerio de Salud, la Universidad, el Colegio Médico de Chile y la Sociedad Chilena de Pediatría.

En reconocimiento de su valioso desempeño profesional, que ha influido favorablemente en la calidad de la formación pediátrica durante la segunda mitad del siglo, el Dr. Meneghello ha recibido importantes distinciones académicas, destacándose entre ellas la de Experto en Educación en Pediatría de la Organización Mundial de la Salud (1960); Miembro de Número de la Academia Chilena de Medicina (1986); Profesor Emérito de Pediatría de la Universidad de Chile (1988); Condecoración de la Orden de la
Cruz del Sur, del Ministerio de Salud de Chile (1989) y Premio de Educación Superior de la Universidad Santo Tomás (1995).

En el segundo semestre del año en curso dichos homenajes culminan con el otorgamiento de la Medalla "Rector Juvenal Hemández Jaque" (septiembre 1995) -n atención a su ejercicio profesional sobresaliente- y la designación por la American Academy of Pediatrics, durante la Reunión Anual celebrada en la ciudad de San Francisco, para el premio "Medical Education Lifetime Achievement Award", en atención a su vida académica de excelencia.

Los logros alcanzados por el Profesor Julio Meneghello han recibido siempre el valioso estímulo espiritual de su distinguida esposa, la Dra. Ana María Ester Torti Lobos, y de sus hijos Marcelo, Pedro y Claudia.

Para nosotros, sin duda, ha constituido una oportunidad muy valjosa, que agradecemos, el haber participado -junto a muchos de sus colaboradores- desde el inicio de la carrera profesional que reseñamos integramente dedicada a la Universidad y a la Pediatría. 